\title{
Drug Induced Methemoglobinemia
}

\section{Cobas, H. Olivera, G. Soto, T. Fuhrman}

Department of Anesthesia and Perioperative Medicine, University of Miami Miller School of Medicine, University of Miami Hospital, Miami, USA.

Email: tfuhrman@med.miami.edu

Received March 14 ${ }^{\text {th }}, 2013$; revised April 18 ${ }^{\text {th }}, 2013$; accepted May 6 ${ }^{\text {th }}, 2013$

Copyright (C 2013 M. Cobas et al. This is an open access article distributed under the Creative Commons Attribution License, which permits unrestricted use, distribution, and reproduction in any medium, provided the original work is properly cited.

\begin{abstract}
The normal presentation of a patient with a drug induced methemoglobinemia is a low pulse oximetry $\left(\mathrm{SpO}_{2}\right)$ reading, usually in the mid $80 \%$ range, while having a very high $\mathrm{PaO}_{2}$ on an arterial blood gas (ABG)1. We present a case where the initial ABG showed a very high $\mathrm{PaO}_{2}$ and a metabolic alkalosis while the $\mathrm{SpO}_{2}$ fluctuated $(85 \%-99 \%)$. Those findings combined with hemodynamic instability complicated the diagnosis and delayed optimal care.
\end{abstract}

Keywords: General Anesthesiology Clinical Anesthesiology Anesthetic Monitoring

\section{Introduction}

The normal presentation of a patient with a drug induced methemoglobinemia is a low pulse oximetry $\left(\mathrm{SpO}_{2}\right)$ reading, usually in the mid $80 \%$ range, while having a very high $\mathrm{PaO}_{2}$ on an arterial blood gas (ABG) [1]. We present a case where the initial ABG showed a very high $\mathrm{PaO}_{2}$ and a metabolic alkalosis while the $\mathrm{SpO}_{2}$ fluctuated (85\% - 99\%). Those findings combined with hemodynamic instability complicated the diagnosis and delayed optimal care.

\section{Case Report}

The patient was a 70-year-old African American male who presented with a diagnosis of multinodular goiter. He was schedule to undergo a complete thyroidectomy, via a transcervical approach for substernal component. Past medical and surgical history included the following: implantation of a pacemaker/Automated Internal Cardiac Defibrillator (AICD) for Sick Sinus Syndrome and ventricular tachycardia/ventricular fibrillation, hypertension, glaucoma, BPH, diabetes, arthritis, chronic renal insufficiency, aortic valve replacement, bilateral cataract surgery, partial thyroidectomy, appendectomy and a bowel resection for gastrointestinal bleeding with obstruction. The patient had also been diagnosed with obstructive sleep apnea (OSA), and was using Continuous Positive Airway Pressure at night. Preoperative laboratory values are listed in Table 1.

The anesthetic plan was for a general endotracheal an- esthetic. After surgery, following recovery from anesthesia in the Post Anesthesia Care Unit (PACU), the patient was to be transferred to the Surgical Intensive Care Unit (SICU) for close observation because of his cardiac condition and his OSA.

The initial anesthetic and surgery were accomplished without complications. Induction and endotracheal intubation were noted as uneventful; laryngoscopy resulted in direct vision of the vocal cords. Surgery proceeded without incident and the goiter was dissected and removed. The patient's trachea was extubated and after the patient spent his initial recovery in the PACU he was transferred to the SICU.

On the first post-operative day, while still in the SICU, the patient began to develop an expanding hematoma. The decision was made to bring the patient back to the operating room. The hematoma was evaluated as to not be compromising the patient's airway. Anesthesia induction was again uncomplicated and intubation was accomplished by direct laryngoscopy. It was noted that the vocal cords did appear to be somewhat edematous.

The surgical incision was opened and the wound drained. There was no apparent arterial bleed, just gen-

Table 1. Preoperative data.

\footnotetext{
Hct 46.3, Hgb 16.1, Plts 204 k, WBC 7.9

$\mathrm{Na}^{+} 140, \mathrm{~K}^{+}$4.8, $\mathrm{CO}_{2}$ 31, Cl 105, BUN 19, Cr 1.39, Glu 106

PT: 13.6, PTT 31, INR 1.3

EKG: SR with Left axis deviation, RBBB
} 
eral oozing. The incision was closed with staples, and a drain placed. The trachea was extubated and the patient transported to PACU for recovery. After stabilization he was again transferred to the SICU.

Two days later the patient was urgently returned to the operating room for the second time due to another expanding hematoma. This time, however, while the patient was alert and cooperative, the hematoma was clearly impinging on the trachea. The plan was for an awake fibreoptic intubation. The patient was pre-oxygenated and oral topical anesthesia was achieved with topical benzocaine $20 \%$ (hurricane spray). The hurricane spray was applied three times. The oxygen saturation $\left(\mathrm{SpO}_{2}\right)$ was maintained at $100 \%$ during the attempts at fiberoptic intubation but since the hematoma was obviously still expanding, the decision was quickly made to perform a rapid sequence induction and intubation utilizing a video laryngoscope. The intubation was successful and confirmed by end tidal $\mathrm{CO}_{2}$ and bilateral breath sounds. Shortly after the surgeons started to evacuate the hematoma, the patient's $\mathrm{SpO}_{2}$ 's were noted to be in the range of $80 \%-86 \%$ in spite of unchanged end tidal $\mathrm{CO}_{2}$ and breath sounds. Blood pressure was 110/60 and the heart rate in the 80's. The $\mathrm{SpO}_{2}$ probe was changed to another finger and then another probe was tried with the saturations still in the 80 's. However before more could be done, the blood pressure (BP) dropped severely (lowest 45/20). Intravenous fluid and phenylephrine boluses were given and the BP responded to 75/40. A norepinephrine infusion was started and titrated to keep the BP systolic above 90 . An ABG was sent to the stat lab. The ABG had a $\mathrm{PaO}_{2}$ of 596 (see Table 2). That should have allowed the clinicians to make a preliminary diagnosis of methemoglobinmeia however the $\mathrm{SpO}_{2}$ 's during this event ranged from $85 \%$ - 99\% which truly confused the issue.

After quickly completing the surgical procedure, the

Table 2. ABG results.

\begin{tabular}{cc}
\hline \multicolumn{2}{c}{ ABG in OR after saturation fell } \\
\hline $\mathrm{pH}$ & 7.42 \\
$\mathrm{PaCO}_{2}$ & 47 \\
$\mathrm{PaO}_{2}$ & 596 \\
$\mathrm{BE}$ & +7 \\
$\mathrm{HCO}_{3}$ & 30 \\
$\mathrm{MetHb}$ & Not available \\
$\mathrm{Pulse}$ & $80 \%-86 \%$ \\
\multicolumn{2}{c}{$\mathbf{A B G / C o - o x i m e t r y ~ i n ~ I C U ~}$} \\
$\mathrm{pH}$ & 7.50 \\
$\mathrm{PaCO}_{2}$ & 32 \\
$\mathrm{PaO}_{2}$ & 369 \\
$\mathrm{BE}^{2}$ & +2 \\
$\mathrm{HCO}_{3}$ & 25 \\
$\mathrm{MetHb}^{\mathrm{O}}$ & $40 \%$ \\
$\mathrm{Pulse}^{2}$ & $94 \%$ \\
& Oximetry
\end{tabular}

patient was transported to the Intensive Care Unit. He remained intubated and sedated now requiring minimal vasopressor support. Upon arrival in the ICU the patient was starting to follow commands and had a $\mathrm{SpO}_{2}$ of $94 \%$. An ABG was sent from the ICU, this time it was also sent for hemoximetry analysis. The results were a $\mathrm{PaO}_{2}$ of 369 and a methemoglobin (metHb) level of 40\%, (normal $0.6 \%$ - 20\%) (see Table 2). The results had just returned and the treatment of methemoglobinmeia was being discussed when the patient extubated himself and very quickly went into cardiac arrest. In spite of immediate implementation of Advanced Cardiac Life Support protocols the patient could not be revived.

\section{Discussion}

Previously the diagnosis of methemoglobinemia depended on clinical suspicion [2] that was confirmed by a hemoximetry analysis. Now the standard use of pulse oximetry has often led to an earlier diagnosis. Clinicians faced with a low pulse oximeter reading and searching for a cause will often send an arterial blood sample for analysis. The $\mathrm{PaO}_{2}$ on a sample from a patient with methemoglobinemia will be very high and certainly not in line with the pulse oximeter values. If that blood sample was not analyzed for hemoximetry, a second sample is then usually sent immediately to the lab for the definitive diagnosis. Newer models of pulse oximeters with multiwavelength technology enable clinicians to diagnose methemoglobinemia as it occurs [3].

This patient presented with some of the classical signs of methemoglobinemia after treatment with topical benzocaine; the $\mathrm{SpO}_{2}$, with readings in the 80's seemed almost diagnostic of methemoglobinemia. Yet the $\mathrm{SpO}_{2}$ did not stay in the 80's and was as high as $99 \%$ before leaving the operating room and $94 \%$ when the ABG was drawn in the ICU.

In almost all case reports describing methemoglobinemia, the patients involved exhibit low $\mathrm{SpO}_{2}$ 's in spite of an enhanced $\mathrm{FiO}_{2}$. An ABG was often obtained (sometimes the blood looked brown) and the resultant $\mathrm{PaO}_{2}$ was always out of line with the pulse oximeter reading, thus basically diagnostic for methemoglobinemia. Our search did not reveal any similar incidences of the $\mathrm{SpO}_{2}$ rising towards normal values before treatment had been undertaken. Nor were there many reports of severe hemodynamic instability associated with methemoglobinemia. Perhaps the critical condition of our patient somehow resulted in incomplete mixing of his deoxygenated (methemoglobin) and some oxygenated blood that resulted in the changing $\mathrm{SpO}_{2}$. The severe hypotension and subsequent vasopressor treatment certainly could have reduced blood flow to all but the vital organs. Then the reperfusion after resuscitation resulted in blood returning to the lungs to be 
oxygenated. However this blood could have been very acidotic. The acidosis then could have triggered the final event of cardiac arrest (especially in a weakened heart that has just suffered a significant insult).

This case illustrates the importance of investigating differential diagnoses when faced with monitors and laboratory findings that that do not fit the clinical circumstances. Given the initial ABG with a low $\mathrm{SpO}_{2}$ especially after the use of benzocaine spray we can only speculate that a more rapid clinical suspicion and treatment of the methemoglobinemia could have precluded some of the hypotension, the need for the postoperative ventilation and the dire consequences that followed.

\section{REFERENCES}

[1] S. J. Barker, K. K. Tremper and J. Hyatt, "Effects of Methemoglobinemia on Pulse Oximetry and Mixed Venous Oximetry,” Anesthesiology, Vol. 70, No. 1, 1989, pp. 112 117.

[2] J. Guay, "Methemoglobinemia Related to Local Anesthestics: A Summary of 242 Episodes," Anesthesia Analgesia, Vol. 108, No. 3, 2009, pp. 837-845.

[3] E. Annabi and S. J. Barker, "Severe Methemoglobinemia Detected by Pulse Oximetry,” Anesthesia Analgesia, Vol. 108, No. 3, 2009, pp. 898-899. 